\title{
Diacronie
}

Studi di Storia Contemporanea

$N^{\circ} 17,1 \mid 2014$

Periferie. Cultura, economia, politica

\section{«A Muvra», 1920-1939: la caricatura in Corsica}

\section{Marie-Claude Lepeltier}

Traduttore: Jacopo Bassi

\section{(2) OpenEdition}

\section{Journals}

\section{Edizione digitale}

URL: http://journals.openedition.org/diacronie/998

DOI: $10.4000 /$ diacronie. 998

ISSN: 2038-0925

\section{Editore}

Association culturelle Diacronie

\section{Notizia bibliografica digitale}

Marie-Claude Lepeltier, « «A Muvra», 1920-1939: la caricatura in Corsica », Diacronie [Online], № 17,

1 | 2014, documento 2, Messo online il 01 mars 2014, consultato il 01 mai 2019. URL : http://

journals.openedition.org/diacronie/998; DOI : 10.4000/diacronie.998 


\title{
Diacronie
}

\section{2/}

\section{«A Muvra», 1920-1939: la caricatura in Corsica}

\author{
Marie-Claude LEPELTIER * \\ traduzione di Jacopo BASSI
}

La fine del XIX secolo vede una moltiplicazione dei movimenti nazionalisti in tutte le aree europee. In Corsica, in questo periodo emerge una corrente autonomista. Nel 1923 Petru Rocca, ispirandosi al Partito Sardo d'Azione, crea il Partitu Corsu d'Azione che assume successivamente la denominazione di Partitu Corsu Autonomista. I dirigenti affermano di non essere separatisti. Questo partito, nato in seno a una cerchia di intellettuali, denuncia il sistema politico francese quale causa dei problemi dell'isola e difende la lingua e la cultura corsa. Il giornale politico «A Muvra», fondato nel 1920 - e parzialmente redatto in lingua corsa - funge da tribuna del PCA. Nel corso degli anni il contenuto politico del giornale si fa più circostanziato fino ad approdare, nel 1927 ad una definizione dell'autonomia. Si batte per difendere gli interessi della Corsica lottando contro la "francesizzazione" forzata; per raggiungere le campagne il giornale pubblica spettacoli teatrali e letteratura in lingua corsa, ma, al contempo, inserisce in prima pagina una vignetta, il cui compito è di raggiungere il grande pubblico: ad essa è affidato il compito di sintetizzare il pensiero dei corsisti.

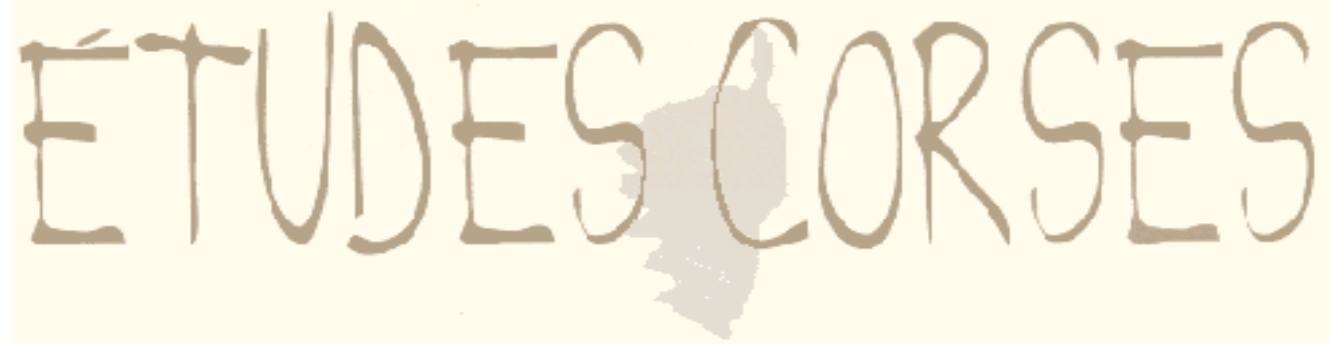

Il presente articolo viene pubblicato in traduzione originale italiana per gentile concessione della rivista Études Corses. 


\section{Introduzione}

«La caricature est l'art de déformer une image pour en faire un tableau plus vrai»1.

Ronald Searle

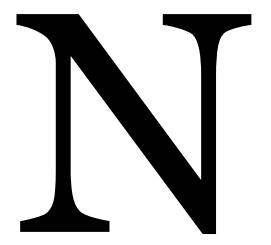

el periodo tra le due guerre la caricatura, che fino ad allora era stata impiegata principalmente dalla stampa specializzata, diviene appannaggio della pubblicistica rivolta ad un pubblico più ampio, spesso, per riprendere il giudizio di Ketty Pierrot $^{2}$, a scapito della sua forza polemica. Più precisamente, il termine "caricatura" deriva dal verbo "caricare", che significa esagerare. Secondo il Grand dictionnaire encyclopédique Larousse, si tratta di «une représentation grotesque, en dessin, en peinture, etc., obtenue par l'exagération et la déformation des traits caractéristiques du visage ou des proportions du corps, à des fins nettement satiriques». Nel primo dopoguerra, il termine evolve e assume il significato di vignetta velata da note umoristiche o satiriche ${ }^{3}$. È dunque un medium abitualmente utilizzato dai giornali d'opinione.

«A Muvra», nella sua veste di periodico di propaganda, ha il profilo ideologico (e i mezzi finanziari4) per utilizzare la caricatura come arma politica: corrisponde allo spirito del settimanale che «20 ans durant mena le combat du réveil et de la révolte insulaire»5. Apparso tra il 1920 e il 1939, "A Muvra» è l'organo di stampa dei corsisti e del loro partito, il PCA (Partitu Corsu d'Azione), creato nel 1922 e divenuto PCA (Partitu Corsu Autonomista) nell'ottobre 1926. Secondo Hyacinthe Yvia-Croce, «Etre corsiste, c'est en un mot, vouloir ardemment demeurer corse, vivre et agir en Corse, par et pour la Corse» ${ }^{6}$. Seguendo le orme di Santu Casanova e del suo «A Tramuntana»7,

\footnotetext{
${ }^{1}$ SEARLE, Ronald, La caricature, art et manifeste: du 16. siècle à nos jours, Généve, A. Skira, 1974, p. 3 .

2 PIERROT, Ketty, La caricature en France de 1919 à nos jours, Mémoire IEP, Aix Marseille 3, 1995.

3 GEORGI, Franck, TOUATI, François-Olivier, «Le dessin de presse et les dessinateurs: des objets d'étude pour l'historien» in Sources: travaux historiques, 26, 1991, pp. 69-80.

4 POLACCI, Daniel, Les autonomistes corses de l'entre-deux-guerres, Mémoire de Maîtrise, Aix en Provence, 1974.

5 YVIA-CROCE, Hyacinthe, Vingt années de corsisme: chronique corse de l'entre-deux-guerres, Cyrnos et Méditerranée, 1979, p. 8.

6 Ibidem, p. 14.

7 Il settimanale «A Tramuntana» fu edito dall'11 ottobre 1896 al 23 ottobre 1919; nato per iniziativa di Pierre-Toussaint Casanova aveva una connotazione politica, umoristica, satirica e letteraria ed era interamente redatto in lingua corsa. Fu espressione del regionalismo isolano portando avanti la denuncia delle condizioni di arretratezza economica, sociale e politica della Corsica. PELLEGRINETTI, Jean-Paul, ROVERE, Ange, La Corse et la République. La vie
} 
«A Muvra» è redatto in gran parte nella langue du maquis: la difesa della lingua corsa è una delle priorità dei corsisti. Il periodico vede la luce a Parigi, al termine della prima guerra mondiale. L'evento bellico, favorendo l'incontro della popolazione francese, il confronto di tutte le identità regionali francesi, è all'origine della presa di coscienza dell'esistenza di un'identità corsa e di valori corsi, che, per i corsisti, occorre difendere da una francesizzazione giudicata indebita. È necessario ricordare che il fondatore della rivista, Petru Rocca, viveva proprio a Parigi quando ebbe l'idea di creare il periodico: era pertanto osservatore attento della stampa parigina che aveva a disposizione e che leggeva certamente tutti i giorni. Aveva allora potuto misurare l'impatto che può avere un disegno sulla coscienza collettiva e sull'opinione pubblica. Si può dunque supporre che non potesse immaginare un tipo di stampa diversa da quella illustrata, soprattutto per un giornale di propaganda. In effetti, malgrado le difficoltà tecniche (la prima zincografia sull'isola viene aperta solo dopo la seconda guerra mondiale) e il costo finanziario che questa implica, la redazione di «A Muvra» si prende l'incarico di pubblicare uno o, a volte, due disegni per numero; questo per via del fatto che la caricatura non è una semplice illustrazione umoristica: proprio perché si nutre dell'attualità immediata, informa, testimonia, ma, soprattutto interpella. Il suo obiettivo è di suscitare una reazione: svolge una funzione impressiva ${ }^{8}$.

Dopo uno studio sulle caricature pubblicate dal periodico una considerazione si impone: queste sono, da sole, bastanti a illustrarne la linea. Non è indispensabile leggere gli scritti per comprendere il pensiero dei corsisti, il che ci permette di misurare il primato dell'immagine sul testo9. L'utilizzo della caricatura si traduce qui in termini di necessità; proprio perché il disegno è un linguaggio universale e polisemico, si indirizza a un pubblico più vasto: anche gli illetterati possono avervi accesso. È più facile ricordare un disegno - che riassume una situazione, un'idea - che un articolo.

L'illustrazione è dunque un elemento essenziale della capacità attrattiva della stampa; la sua influenza sull'opinione pubblica risulta fondamentale; a riprova di ciò la tipografia di «A Muvra» pubblica a partire dal 1923 «l'Almanaccu di A Muvra» successivamente divenuto «U Librone di A Muvra» nel 1937. Sotto la direzione di Daniel Polacci il giornale edita 1.200 copie nel 1924 e il numero dei suoi abbonati è di 806; successivamente divengono 504 nel 1928 e l'almanacco - che ha una tiratura di

politique de la fin du second Empire au début du XXIe siècle, Paris, Seuil, 2004, pp. 233-239 [n.d.t.].

8 SERRE-FLOERSHEIM, Dominique, Quand les images vous prennent au mot: ou comment décrypter les images, Paris, Ed. d'organisation, 1993, p. 37.

9 Durante la Terza Repubblica si giunse al punto per cui la censura rifiutava un disegno ma ne accettava la sua versione scritta, ovvero la descrizione del disegno e della sua didascalia. 
3.500 copie nel 1930 - viene venduto a un prezzo molto contenuto ( 8 franchi): è infatti destinato a diffondere le idee corsiste in ogni angolo dell'isola. Qui viene ripreso l'insieme delle caricature edite e a queste ne vengono aggiunte di nuove. Per ogni rivista (o almanacco) acquistato, quanti lettori - o, si potrebbe dire uditori, nei villaggi - si possono stimare? Non era infatti necessario acquistare la rivista per far cadere l'occhio sulla vignetta, generalmente inserita nella prima pagina: «l'image doit être vue avant d'être lue» ${ }^{10}$.

Le caricature erano realizzate da giornalisti, ma questi erano prima di tutto militanti convinti. Facendo leva su alcuni simboli, denunciavano la situazione economica e politica dell'isola, suggerendo - come se si trattasse di un'evidenza - la soluzione al problema corso: l'autonomia.

\section{Le caricature corse}

Nell'arco di diciannove anni la rivista ha pubblicato circa 500 caricature, contrassegnate da sole ventuno firme differenti, il che non significa necessariamente ventuno disegnatori diversi. Sembra che soltanto cinque disegnatori abbiano realmente contribuito alla rivista, quasi a confermare la teoria secondo la quale è più difficile improvvisarsi disegnatori piuttosto che giornalisti, almeno per lo spirito giornalistico dell'epoca. Il primo disegnatore a intervenire è Matteo Rocca (1896-1955), il fratello di Petru Rocca; questi è anche capo redattore e firma numerosi articoli celato sotto diversi pseudonimi (Petroniano, Egomet, ...). Fino al 15 giugno 1921, Rocca è il solo disegnatore e il suo nome si rinviene regolarmente fino al 1923, per poi eclissarsi tra il 1924 e il 1928. Ritorna in forze nel 1929, con 23 disegni in quello stesso anno. A partire dal 1932 e fino alla soppressione della rivista, nel 1939, è l'unico disegnatore. Autore di circa 300 caricature, ossia più della metà, è, secondo L'Anthologie des écrivains corses: «un peintre de talent, un caricaturiste mordant et spirituel, et en cette qualité membre exposant à la société des beaux-Arts de Nice [...]» ${ }^{11}$. Valutare l'aspetto qualitativo di un disegno, quale che sia - operazione che prospetta un giudizio di valore, ossia di parlare dell'aspetto estetico di una caricatura politica - è decisamente più difficile e non ci preme. Come sottolinea Jean-Baptiste Carpentier, l'immagine politica è un «messaggio per altri» e deve pertanto essere concepita, redatta e diffusa pensando costantemente al

10 CARPENTIER, Jean-Baptiste, «L’image fixe dans la communication politique», in Humanisme et entreprise, 3/1981, p. 29.

${ }^{11}$ YVIA-CROCE, Hyacinthe, Anthologie des écrivains corses, t. 3, Ajaccio, Ed. Cyrnos et Méditerranée, 1987, p. 196. 
suo lettore sapendo che questi non è un richiedente del messaggio ${ }^{12}$. Se è vero che Matteo Rocca è un caricaturista «mordant et spirituel», il suo disegno, un po' goffo all'inizio, col tempo si consolida. I tratti divengono più sicuri, più precisi: evidentemente ha preso gusto nel tratteggiare le sue caricature. Non si fissa su un solo stile grafico. Artista dei suoi tempi, è aperto al mondo e ispirato dalle diverse correnti artistiche che animano il periodo fra le due guerre (il cubismo, ma anche l'influenza dei surrealisti). Questo periodo conosce un cambiamento nella formazione artistica dei disegnatori: «Ce ne sont plus des artistes peintres mais des journalistes qui s'expriment par le dessin»13. Matteo Rocca è dunque una figura intermedia fra queste due professioni; ma non ci si può ingannare sul fatto che fosse anzitutto un militante corsista. Aderisce completamente alle idee del movimento, ne è al contempo uno dei pilastri e mette il suo talento e la sua matita al servizio della causa.

Un secondo disegnatore, Kryn, ossia Dominique Massa ${ }^{14}$, gioca un ruolo essenziale. Pur essendo presente dal 1921 sotto diversi pseudonimi, è il principale disegnatore tra il 1924 e il 1928. Vanta al suo attivo più di 155 disegni ed è al contempo segretario di redazione e autore di alcuni articoli. Disegnatore di talento, con la sua matita esprime sentimento ed emozione: trova le espressioni idonee a riassumere le situazioni e sa essere d'effetto. Il suo tema prediletto è l'esilio dei Corsi: apparentemente è la cosa che ha più a cuore e che lo ispira maggiormente. Tratteggia un personnaggio presuntuoso e ridicolo: Canistro, un corso espatriato ${ }^{15}$. Niente sfugge al suo spirito caustico. Constatiamo che quando utilizza uno dei nomi fittizi (Ranfia, Vespa e Urticula, nomi che sono tutto un programma...) il contenuto dei suoi disegni risulta più incisivo, più impegnato politicamente; si firma infatti anche sotto il nome di Khi, EU, KS o Mangarelli.

Un altro disegnatore molto attivo tra 1923 e 1925, ma dotato di uno stile radicalmente differente è A. Dell Pellegrino, detto Toto, riconoscibile per alcuni ritratticaricatura che rappresentano essenzialmente i suoi concittadini aiaccesi. L'obiettivo è unicamente il divertimento. I tratti sono precisi, il disegno è spoglio, limitato all'essenziale. Kyrn si è a sua volta interessato a questo genere d'esercizio di stile allorquando ha disegnato alcuni uomini politici corsi (Célestin Caïtucoli, Vincent De Moro-Giafferi...). Entrambi abbozzano il ritratto dei principali collaboratori della rivista

${ }^{12}$ CARPENTIER, Jean-Baptiste, «L'image fixe dans la communication politique», in op. cit., p. 27 13 DUCCINI, Hélène, «La caricature politique», in Historia, 251, 2001, p. 67.

14 È autore anche di alcune caricature apparse su «Corsica».

15 Kyrn ne fa una sapida descrizione in un articolo comparso su «A Muvra» nel 1927. 
(ill. 1). È un modo per mettere un viso su un nome, di associare il lettore alla grande famiglia dei corsisti: non essere più anonimi è un po' come essere amici.

J.A. Geromini e P. Pollo, invece, firmano insieme 14 disegni tra il 1924 e il 1927; ma sono proprio questi disegni a risultare particolarmente importanti dal momento che il loro contenuto è molto forte. Il loro universo è cupo e l'umorismo assente: nei loro disegni si riscontra una discreta aggressività.

L'immagine della loro isola, ma anche il messaggio del P.C.A. - che i vignettisti trasmettono attraverso i loro disegni - sono affidati essenzialmente al talento e all'interpretazione dei due disegnatori, addirittura ad uno solo a partire dal 1932. Possiamo interrogarci sul valore delle testimonianze ma, in tutti i casi, se la rivista accetta di pubblicarle, queste corrispondono allo spirito della rivista e dunque allidea che si vuole dare della Corsica. Come ha scritto Jacques Lethève: «[...] un dessinateur satirique n'est pas un isolé»16. Questi disegni riflettono lo stato d'animo di una minoranza; ma è una minoranza di cui non è possibile negare l'esistenza e, dal momento che si esprime, è una minoranza attiva. Malgrado si tratti di disegni di propaganda, sono una parte della narrazione storica. È una caricatura etnocentrata che permette di scoprire i disagi della società. Michel Ragon osserva, correttamente, che «le chauvinisme semble avoir lié un pacte avec la caricature» ${ }^{17}$ : si indirizza a un pubblico mirato.

\section{Simboli}

Per essere efficace, una caricatura deve risultare comprensibile a tutti. Un disegno si deve rivolgere a un pubblico che condivida gli stessi riferimenti storici e culturali, necessari per comprendere le allusioni e gli altri giochi di parole. Per realizzare questo i caricaturisti utilizzano codici e simboli. Ogni situazione, ogni personaggio, deve essere chiaramente identificabile, senza rischiare di ingenerare errori o confusione; a ciascuno viene attribuito il proprio attributo: alla feluca corrisponde il prefetto e così via.

Parallelamente ai codici in uso, i vignettisti hanno utilizzato simboli che sono per loro peculiari: così, a partire dal 1921, Kyrn (Ranfia) mette in scena un muflone.

\footnotetext{
${ }^{16}$ LETHÈVE, Jacques, La caricature sous la IIIè République, Paris, A. Colin, 1986, p. 77.

${ }_{17}$ RAGON, Michel, Le dessin d'humour: histoire de la caricature et du dessin humoristique en France, Paris, Seuil, 1992, p. 58.
} 


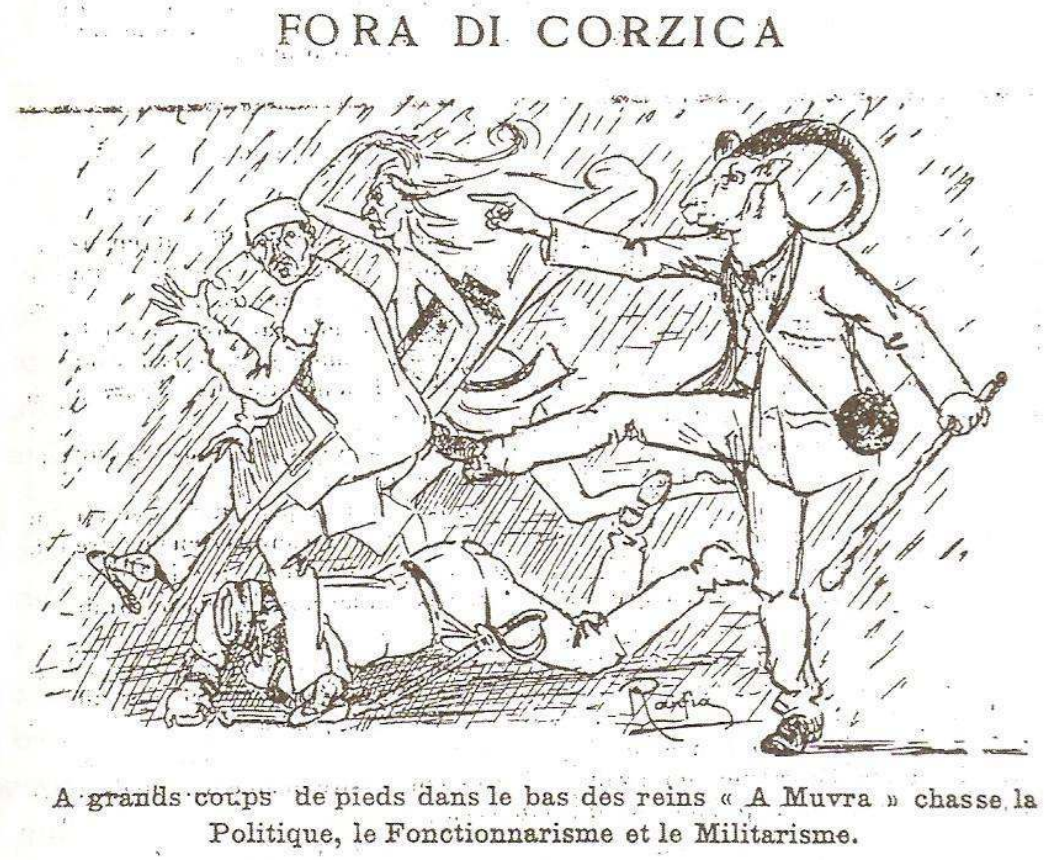

Ranfia, A Muvra, 22 septembre 1921.
Secondo Bertrand Tillier la metamorfosi è un metodo di destituzione simbolica frequentemente utilizzato dai caricaturisti: «par exemple en représentant des régressions de l'humain vers le stade animal, végétal ou monstrueux»18. I disegnatori hanno

fatto ricorso a questo procedimento, invertendolo, con l'obiettivo di valorizzarlo. Petru Rocca spiega che vedendo un muflone corso dimenarsi tra le barriere di una gabbia, allo zoo, ha avuto l'idea del nome "A Muvra» per la sua rivista; secondo Paul Simonpoli, questo animale è: «symbole de pureté, d'innocence, de fragilité, de maternité, [il] représente un idéal féminin»19; "est rebelle à la domestication, à la tutelle, à la soumission. Et même quand il est capturé, il sait garder la maîtrise de son destin ${ }^{20}$. È proprio ciò di cui si tratta. I vignettisti, ispirandosi al titolo della rivista e al suo potere simbolico, hanno ripreso per conto proprio il tema del muflone facendone un eroe dalle sembianze umane. Nei primi disegni il muflone rappresenta la rivista in quanto vettore ideologico che è depositario della verità. Permette il risveglio delle coscienze e contribuisce alla lotta contro la francesizzazione indebita dell'isola; ma, dal 1924, il muflone diventa l'emblema della lotta della Corsica per la sua autonomia. I vignettisti hanno associato la rivista e la lotta per una Corsica autonoma in un identico simbolo. Su questo fronte, la rivista, creando coesione, vuole posizionarsi come il portavoce della maggioranza isolana: non bisogna infatti dimenticare che ambisce ad affermarsi come la voce della maggioranza corsa: non bisogna infatti dimenticare che il movimento corsista è minoritario.

18 TILLIER, Bertrand, A la charge! La caricature en France de 1789 à 200o, Paris, Les éditions de l'Amateur, 2005, p. 178.

${ }^{19}$ SIMONPOLI, Paul, «Le mouflon» in SIMONPOLI, Paul (sous la dir. de), La chasse en Corse, Ajaccio, PNRC, 1995, p. 153.

20 Ibidem, p. 168. 
Il secondo simbolo è una donna. Alla maniera di Marianna, che rappresenta allo stesso tempo la Francia e la République, i vignettisti hanno simbolizzato la Corsica come una donna vestita di nero. Così è possibile attribuirle sentimenti, come la collera, l'indignazione, la tristezza o, ancora, il dolore. Questi sentimenti vengono trasmessi al lettore, che sarà ugualmente preda delle stesse emozioni: la forza del disegno risiede in questo. Attraverso il periodico, i corsisti formulano rivendicazioni che si basano sullo stato economico, sociale, culturale e politico della Corsica in un preciso momento storico. Per questo motivo la scelta del vestito di questa donna è così importante: deve possedere attributi che lo pongano al di fuori del tempo. Veste dunque una larga gonna increspata, un corpetto attillato e un grande fazzoletto ( $U$ mandile) nero sulla testa. Le donne erano solite portare abiti lunghi, di colori sobri, mentre le ragazze gonne a fiori. Il simbolo utilizzato è una donna adulta, madre, capace di prendersi cura e di proteggere i suoi figli. I disegnatori tentano di fare prendere coscienza all'opinione pubblica del fatto che, come il simbolo che la rappresenta, la Corsica è capace di prendere in mano il suo destino: rivendica un'autonomia che può gestire.

Matteo Rocca la mette in scena per la prima volta nel gennaio 1921, in un disegno che da solo riassume esaurientemente quanto detto in precedenza. La scena si svolge in un cimitero: qui le tombe sono allineate allinfinito. In primo piano, su una di queste sono deposti un berretto e un elmetto da soldato; una donna seduta, vestita di nero, piange. Su ogni croce è scolpito un nome: i morti non sono anonimi. La didascalia pone l’interrogativo: Parchè? (Perchè?)

$1914-1918$

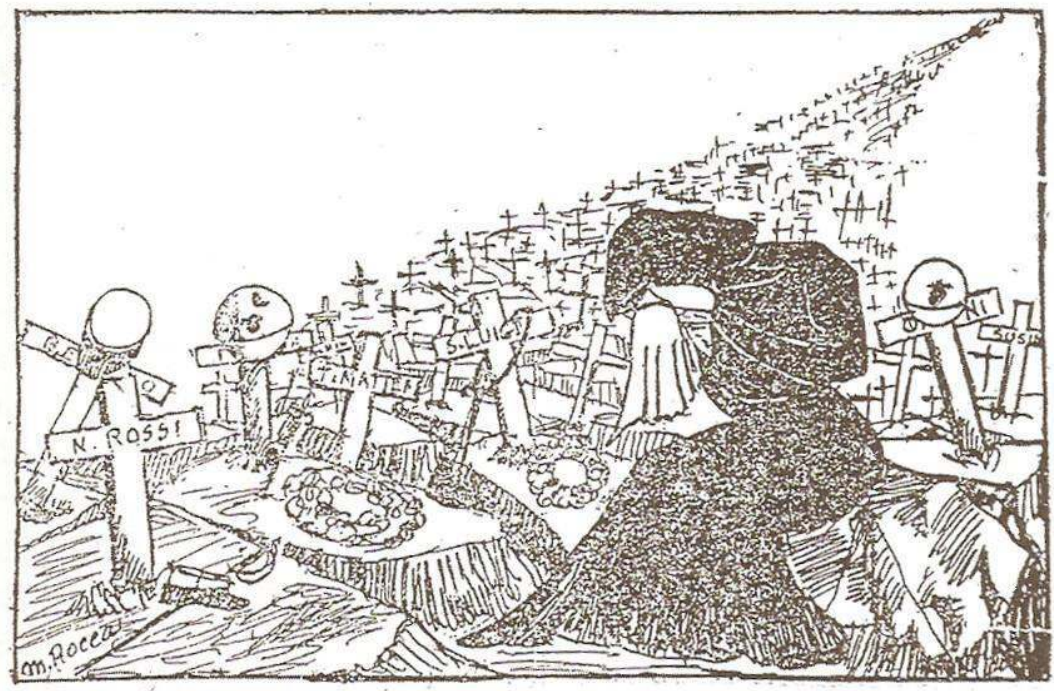

Parche?

Matteo Rocca, A Muvra, janvier 1921.

Il soggetto femminile vestito di nero rappresenta la donna corsa, che è rimasta al "paese" attendendo il ritorno del marito, del fratello, del padre. Allo stesso modo 
rappresenta la Corsica, che piange i suoi figli morti per una causa che forse non era la sua. La Corsica ha contribuito molto alla causa francese in occasione di questa guerra: è assai difficile quantificare il numero reale degli arruolati, dei feriti e degli invalidi di guerra in Corsica. Si tratta comunque di una cifra superiore alla media nazionale: più di 11.300 morti ${ }^{21}$.

Questa immagine femminile, simbolo dell'isola e dei suoi abitanti, è presente in un centinaio di caricature, ossia un disegno su cinque.

Così come i disegnatori si sono serviti di simboli, allo stesso modo hanno utilizzato il tratto grafico. I corsisti sono intimamente convinti dell'esistenza di una razza corsa e s'ingegnano di dimostrare questa idea. Kyrn si trova a suo agio in questo genere d'esercizio.

\section{E DUIE CORSICHE}

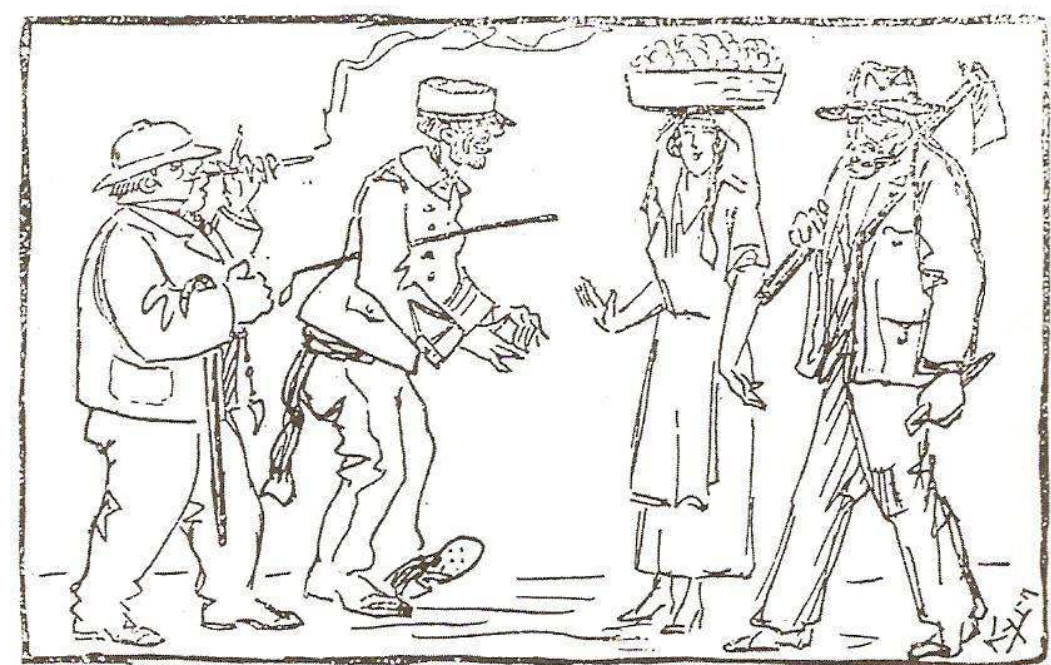

A VERA E L'ALTRA

Kyrn, A Muvra, 1 luglio 1928.

Osservando con attenzione i disegni, possiamo scoprire alcune caratteristiche fisiche attribuite ai Corsi. Nei limiti del talento del disegnatore, gli isolani sono rappresentati belli e robusti e di dimensioni maggiori rispetto agli altri. Per affrontare l'aspro e rigido clima di montagna, la natura ha generato una razza robusta e vigorosa. Gli illustratori non caricano i tratti di questi visi, al contrario di quelli dei continentali o dei compatrioti esiliati. Questi ultimi, a causa del loro "tradimento", hanno smarrito la loro identità: sono rifiutati e ignorati; non possono rivendicare l'appartenenza alla razza corsa, come si evince dai disegni. I Corsi sono belli, contrariamente a tutti gli altri: la bellezza esteriore sottende una bellezza interiore. I vignettisti hanno cercato di

${ }^{21}$ LAHLOU, Raphaël, «Chronique d'une relation difficile: la Corse et la Troisième République (1870-1940)», in BASTIANI, Tony (dir.), Encyclopaedia Corsicae, vol. V, Bastia, Ed. Dumane, 2004, p. 1019. 
eliminare l'immagine del Corso pigro: sono quasi tutti rappresentati alle prese con qualche genere di attrezzo. Nella maggior parte dei disegni gli uomini portano la barba, che sull'isola è segno di lutto. I Corsi hanno quindi i tratti regolari, fermi e volitivi, a immagine e somiglianza del loro comportamento: la caricatura accentua questo lato manicheo. Questo cliché si traspone anche nelle donne; anche se non hanno una tipologia fisica particolare i tratti dei loro visi risultano fermi, regolari e volitivi, espressione di nobiltà d'animo: il loro portamento è altero.

È sufficiente guardare qualsiasi album fotografico dell'epoca o, semplicemente, la galleria di ritratti disegnati da A. Dell Pellegrino - e apparsi su A Muvra - per convincersi del fatto che la realtà fosse tutt'altra.

Difendendo la tesi dell'esistenza di una razza, i corsisti fornivano argomenti per giustificare le loro rivendicazioni: se i Corsi sono una razza a parte, non hanno - né storicamente né biologicamente - nulla a che vedere con i Francesi e, a causa di ciò, vengono ingiustamente sottomessi da loro. Ogni razza ha il diritto di rivendicare per sé la libertà politica, economica e sociale: pertanto questa lotta è giusta. Ma la cosa più importante è preservare la razza, perchè se dovesse scomparire, ne verrebbe rivelata l'inferiorità: è dunque essenziale per i vignettisti definire con precisione le caratteristiche fisiche dei corsi e - laddove possibile - i tratti del loro carattere; ciascuno deve riconoscersi nel modello proposto. I disegnatori suggeriscono che ogni contatto con il continente comporti una degradazione morale o fisica, che lo stesso vignettista si diverte a trasporre visivamente. La caricatura diviene qui sininimo di manipolazione. Beninteso: è necessario prestare attenzione al contesto storico nel quale si situa questa discussione.

Facendo leva su questi simboli i caricaturisti diffondono così il loro messaggio.

\section{Una caricatura propagandistica}

Vengono utilizzati tutti i registri che la caricatura offre: la destituzione simbolica (scatologia, metamorfosi), la parodia, l'ironia, lo humour, l'emozione... Kyrn è più concentrato sui problemi insulari rispetto a Matteo Rocca, che adatta l'attualità nazionale - o internationale - alla visione corsa della situazione.

La lettura delle caricature può essere sviluppata su due livelli.

$\mathrm{Su}$ di un primo piano, noi cogliamo la storia del movimento corsista insieme a quella della rivista in considerazione del fatto che le due realtà risultano legate. «A Muvra» pubblica con regolarità caricature che danno notizia delle diverse manifestazioni culturali o politiche organizzate dal P.C.A. In questo modo, facendone 
partecipe il lettore, i corsisti si rendono accessibili, apportando un tocco di convivialità: cercano di dimostrare che compongono una grande famiglia unita.

Consideriamo, ad esempio,il caso della croce di Ponte Novu, illustrato da Kyrn. Non appena viene lanciata la sottoscrizione per l'erezione di una croce, Kyrn pubblica, nel 1923, un disegno rappresentante il ponte di Ponte Novu davanti al quale una donna vestita di nero - la Corsica - piange perché nessuna croce rende omaggio ai martiri della battaglia dell'8 maggio 1769. Nel 1925 il disegno viene ripubblicato, quasi identico: questa volta, tuttavia, la donna stringe con tutte le forze la croce tanto agognata. Ł̀ un modo per ringraziare i donatori e per festeggiare questo evento, che sa di vittoria. Nello stesso anno viene celebrato il bicentenario della nascita di Pascal Paoli: J.A. Geronimi et P. Pollo rendono omaggio alla sua figura pubblicando un disegno allegorico. Il 14 luglio 1926, una grande festa riunisce i corsisti a Morosaglia, villaggio natale di Pascal Paoli: si tratta della loro terza merendella ${ }^{22}$. L'evento è illustrato da Kyrn²3.

Ma il lettore è compartecipe anche della realizzazione di eventi più schiettamente politici. Così nel 1927 il Partitu Corsu Autonomista si associa ai regionalisti bretoni e alsaziani per creare il Comité central des minorités nationales de France (CCMNF). A partire da questa data le "tre sorelle" - introdotte da un disegno di Matteo Rocca nel 1930 - appaiono con regolarità nelle caricature che vengono realizzate in occasione delle manifestazioni che le vedono protagoniste. Quando le illustrazioni non sono relative a ricorrenze, queste si ergono contro l'ingiustizia e le aggressioni di cui il movimento o il giornale sono vittime.

A titolo di esempio, in occasione dei secondi Stati generali che si svolgono nelle giornate del 27, 28 e 29 agosto 1935 a Bastia, la polizia fa irruzione nella sala. Il 29 agosto, la polizia municipale accompagnata da rinforzi vieta l'accesso alle sale a tutti coloro che non sono muniti della carta di delegato. In seguito a ciò, Matteo Rocca pubblica nell'edizione del 20 ottobre 1935 un disegno intitolato Libertà d'opinione (ill.5). Con esso si sottende che è proprio la nazione corsa tutt'intera ad essere privata della libertà e non i rappresentanti di un partito politico. Per loro, la Corsica è la vittima di un abuso di potere da parte dello Stato francese.

$\mathrm{Al}$ pari della storia del movimento, anche quella del periodico è tormentata: i diversi avvenimenti riferibili alla rivista sono riportati. Sin dalla sua fondazione, la pubblicazione ha destato sospetti nelle autorità. Nel settembre 1923 è oggetto di

${ }^{22}$ La merendella è l'equivalente italiano della scampagnata; nel caso specifico si trattava di ritrovi politici connotati dalla convivialità [n.d.t.].

${ }_{23}^{2}$ Le merendelle successive saranno documentate fotograficamente. 
censura e la sua vendita viene interdetta in Marocco. In seguito, a più riprese, è sotto processo per diffamazione contro «L'Oeuvre» nel 1937, e «La Corse» dal 1929 al 1931. A partire dal 1928, è vittima di una campagna stampa che la accusa di irredentismo. La perquisizione dei locali della rivista, il 3 febbraio 1928, è vissuta come un attentato alla libertà di espressione. Il 10 febbraio 1928, la rivista pubblica un disegno di Kyrn: $A$ Paghiella.

È una maniera per rimarcare che nessuno impedirà al periodico di esprimersi e di continuare la battaglia. Il 3 febbraio 1929, a mo' di commemorazione, viene illustrato il primo anniversario di questa perquisizione. Tuttavia, trascinati dalle loro emozioni, i vignettisti possono essere contradditori: così avviene nel 1931. A seguito di problemi che il giornale ha conosciuto, i disegnatori si lamentano di non potersi esprimere liberamente. Matteo Rocca fa pubblicare un disegno intitolato E liberta di a Stampa. In questo disegno è la Corsica ad essere imbavagliata e non il periodico. Ancora una volta il lettore sussume l'idea che è proprio l'isola intera ad essere ridotta al silenzio: essi s'impongono come portavoce del popolo corso. Sette disegni sul tema della Corsica imbavagliata appaiono dal 1926 al 1939. Per converso, quando vincono il processo che li oppone al giornale «La

INTERRU

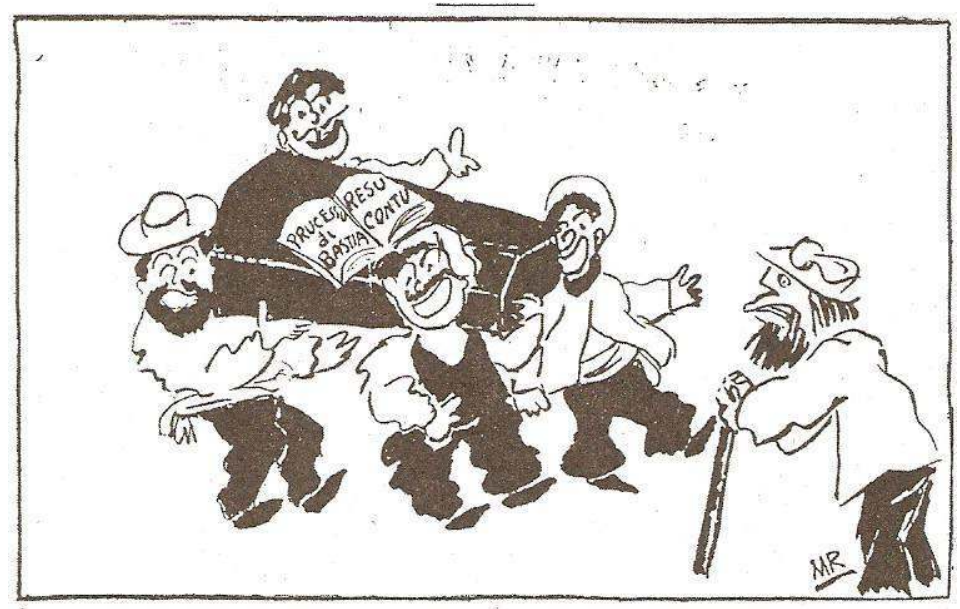

È u corciu di Dumenic' Antone chi voi purtale in 'ssa manera?

Ié! È mortu da a risa, leghiendu l'arringa di Moru contru l'Autonomisti.

Matteo Rocca, A Muvra, 8 marzo 1931.
Corse», sorto in seguito ad una protesta contro una caricatura che tale giornale riteneva diffamatoria, essi si tratta di un trionfo di dimensioni modeste, come è testimoniato dal disegno di Matteo Rocca intitolato Interru.

È la tumulazione del povero Dominique Antoine, deceduto leggendo l'arringa di V. de Moro-Giafferi contro gli autonomisti. «A Muvra» giubila e accomuna il lettore alla sua gioia. La giustizia ha riconosciuto che la rivista poteva esprimersi liberamente. Ma è bene non incorrere in fraintendimenti: questo comportamento, oltre ad una volontà di informare, rientra in un quadro più vasto di propaganda. Si direbbe ai nostri giorni "disinformazione". Ad un secondo livello, noi percepiamo le idee dei corsisti (il contenuto). Come non è evidente il processo che porta ad illustrare un concetto, poiché occorre risalire fino all'essenza 
per toccare il numero maggiore di persone, i vignettisti isolani si occupano di illustrare unicamente le cause e gli obiettivi della loro battaglia. Occorre utilizzare formule ad effetto. Tre temi spiccano: una Corsica povera e abbandonata; una Corsica che si esilia; una Corsica che resiste. La nozione di una Corsica povera è essenzialmente espressa attraverso l'idea di progresso. I disegnatori si sforzeranno di dimostrare che a partire dalla sua integrazione alla Francia nel 1769, l'economia dell'isola va alla malora. Essi denunciano lo stato e il costo dei trasporti, e fanno il paragone con i trasporti sardi che, per loro, sono di migliore qualità e meno cari. Parallelamente a questo, la caricatura denuncia la rivoluzione dei trasporti (maggiore tonnellaggio, rapidità e frequenza) come una delle cause del problema economico: l'isola è invasa da beni di consumo meno cari e di migliore qualità rispetto a quelli prodotti sul luogo e ciò determina un paradosso: «par l'intermédiaire des ports, ce sont les villes qui nourrissent les campagnes» ${ }^{24}$. Allo stesso tempo, se la caricatura ci fa comprendere che la Corsica ha delle possibilità in materia d'agricoltura, soffre volontariamente d'amnesia per ciò che riguarda la qualità dei suoi metodi di produzione e l'arcaismo delle sue tecniche. Non fa menzione degli sfruttamenti parcellari causati dalle divisioni dei terreni in seguito alle successioni e trascura del tutto le clausole doganiere che vessano la Corsica e che, peraltro, la stampa denuncia.

${ }^{24}$ ARRIGHI, Paul (dir.), Histoire de la Corse, Paris, Privat, 1986, 


\section{$\mathscr{A}$ piu Bella}

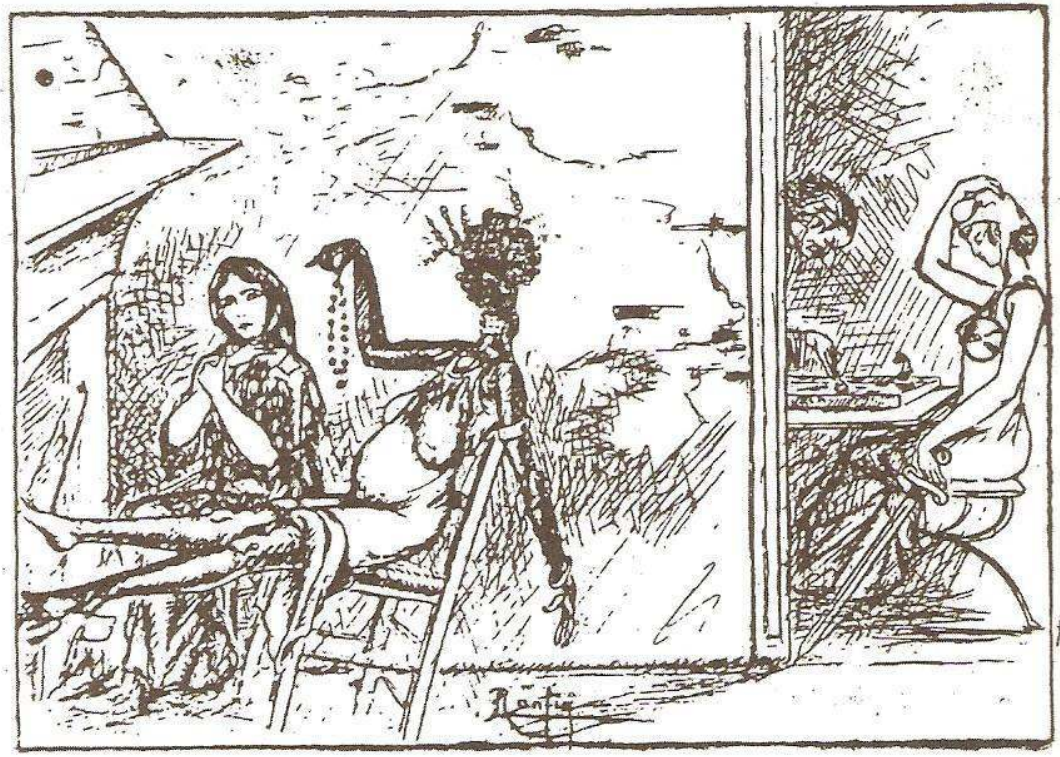

LA CORSE - Comment se fait-1l que la France te donne bijoux, chemins de fer canaux, paqu:bots confortables, aiors qu'elle me laisse čans la plus proiondé misère?

Ranfia, A Muvra, 5 febbraio 1922.

U S.CAPPA-SCAPPA

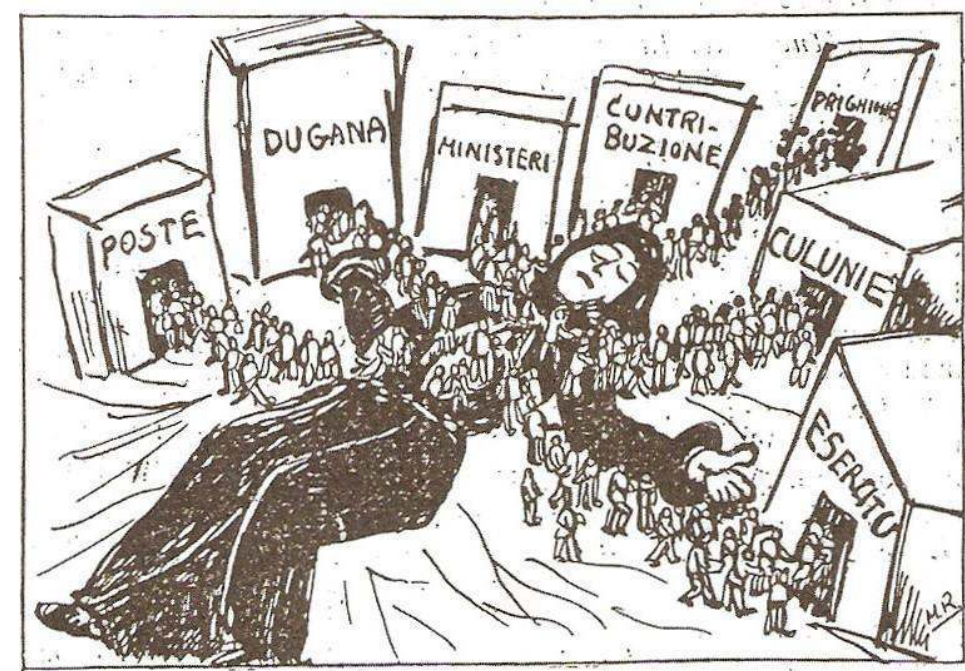

$\because$ CURSIGHELLA DISSANGUATA

Matteo Rocca, A Muvra, 3 gennaio 1932. 
I disegnatori segnalano unicamente ciò che è loro utile per le rivendicazioni: la nozione di progresso passa ugualmente per l'idea di confort (strade, ferrovie, elettricità). In tutti questi settori l'isola accusa un grande ritardo rispetto al continente. Se la caricatura denuncia questo ritardo, ignora gli ostacoli naturali (mare, montagna) che rallentano fortemente i lavori di costruzione delle differenti reti di comunicazione. Più che sulla povertà dell'isola, i disegnatori si focalizzano sull'idea di una Corsica abbandonata dalle autorità francesi: malgrado ciò, nessun disegno qualifica l'isola come dipartimento. L'equivoco è volutamente cercato. L'isola è presentata come una colonia, ma non ottiene dai poteri pubblici la stessa attenzione di una colonia: ancor più che una Corsica dimenticata, tra i cinque disegni dedicati al tema, quattro di questi (uno per anno, dal 1934 al 1937) illustrano una Corsica sconosciuta. Lo humour permette ogni cosa, soprattutto ciò che non può essere scritto. I disegnatori creano e custodiscono un disagio: il lettore non può che ribellarsi di fronte a un'ingiustizia così palese. La causa di questo stato di fatto è, secondo loro, l'incuria del governo, sulla quale insistono, ma anche il clanismo che imperversa sull'isola. Per questo scopo non vengono risparmiati gli uomini politici: Adolphe Landry è particolarmente bersagliato. Tra il 1921 e il 1936, gli vengono dedicati ben ventuno disegni.

Un secondo tema ricorre in modo ossessivo: l'emigrazione dei Corsi e le sue conseguenze. La causa essenziale di questa emorragia è la povertà dell'isola. Più di

Matteo Rocca, A Muvra, 1 giugno 1933.

ATTIVITAI

La crise actuelle préoccupe $\mathrm{N}$. le Prélet, lequel lui consacre le meilleur de son aclivité. I Giurnali.

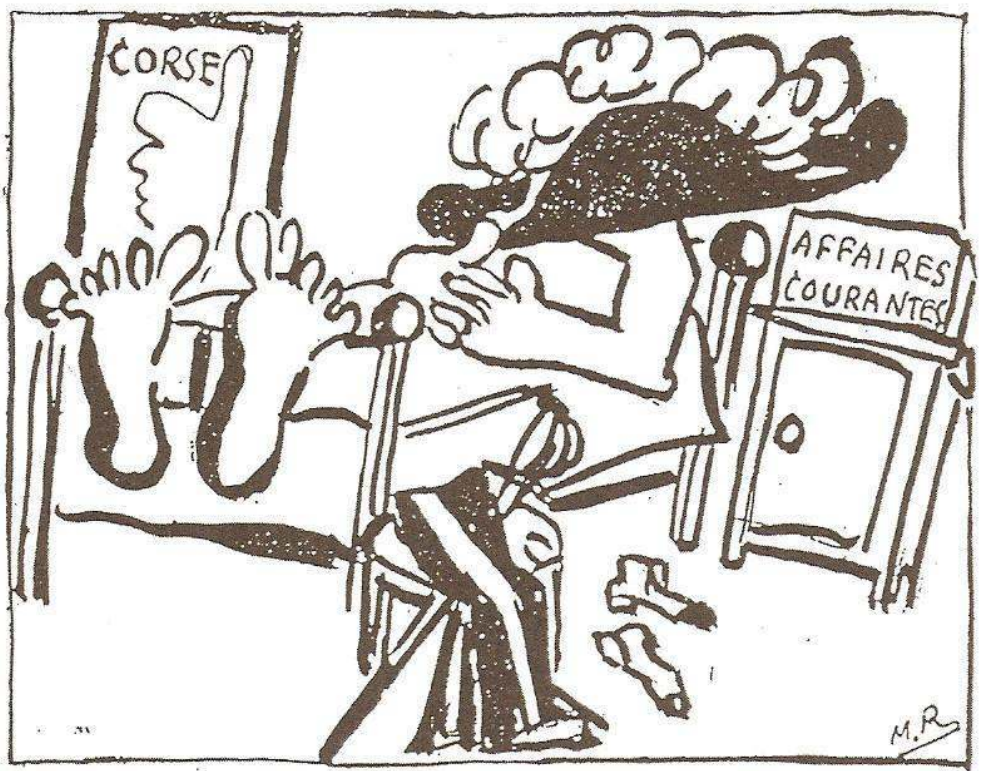

U SGIO PREFETTU A L'OPERA cinquanta disegni hanno un rapporto più $o$ meno stretto con questo tema: qui più che per ogni altro tema i disegnatori utilizzano la carta dell'emozione.Gli illustratori sono Corsi e conoscono bene la mentalità dei loro concittadini: alcuni disegni possono generare senso di colpa. Matteo Rocca prende scientemente come esempio, per una vera 
presa di coscienza, la sepoltura di un padre: che cosa può accadere di peggio a una persona che fare l'ultimo viaggio nell'isolamento più completo, senza figli, senza amici? L'emigrazione massiccia comporta uno spopolamento dei villaggi, con una conseguente destabilizzazione irreversibile della vita sociale ed economica dell'isola. I disegnatori fanno leva sui punti sensibili, in particolare quando illustrano le conseguenze dell'esilio rivolgendosi al lettore. A più riprese Matteo Rocca insiste sul tema dell'invecchiamento degli isolani. Tenta di mettere sull'avviso la popolazione suonando una campana d'allarme, ma si assisterà, ancora nel 1935, a 13.700 partenze, che diverranno 14.800 nel 1937 e 16.700 nel $1939^{25}$.

La vie de nos villoges patil un pet du fuit de licinigration, mais tes Corses ninsi transplantis

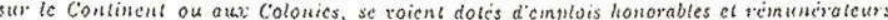

X. Sociolozu corso pitizutu.

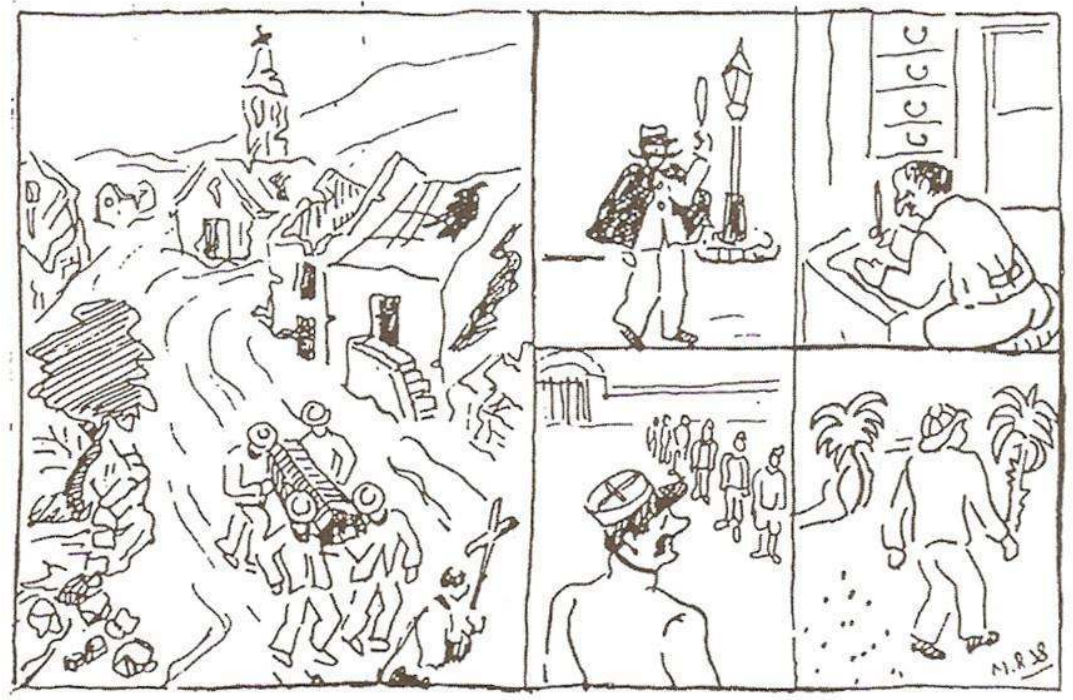

I Quattru Figlioli ch'un ponu accumpagnà u Babbu.

\section{Matteo Rocca, A Muvra, 1 febbraio 1938}

La caricatura ci fornisce una prospettiva preziosissima sul modo in cui i corsisti desideravano che i Corsi percepissero i loro compatrioti che avevano scelto di partire per il continente o le colonie. Emigrazione è sinonimo di funzionario: è per questo che $\mathrm{i}$ disegnatori trattano i Corsi esiliati con disprezzo. In più Kyrn caratterizza i suoi personaggi con sembianze semite: naso grosso e grandi labbra. Un'altra costante caratterizza l'emigrante di ritorno al paese: si crea una distanza rilevante fra ciò che si vuole rappresentare e ciò che è realmente. Nessuno può desiderare di somigliare a questo personaggio grottesco di cui ci si prende gioco. Non tutti i Corsi che sono emigrati sono nella stessa situazione: alcuni di loro hanno fatto carriera in seno all'amministrazione militare, come medici o avvocati; altri hanno avuto successo in

25 Ibidem, p. 415. 
ambito politico. Ma la caricatura ignora completamente quelli che hanno avuto successo: in nessun momento viene evocata questa possibilità. Se, casualmente, si sottende che il successo sia possibile è per insistere sulle conseguenze negative che questo può portare (malattia...). L'impatto di questi disegni non ha conseguito risultati dal momento che l'emorragia demografica è continuata: i Corsi hanno continuato a emigrare. I disegnatori hanno continuato a chiamare in causa i Corsi durante tutto questo periodo, in particolare dal 1929 al 1938, quando uno o due disegni l'anno vengono dedicati a questo tema. Tuttavia i disegnatori non lanciano frecciate verso $\mathrm{i}$ militari, quasi a scusarli. La prima guerra mondiale non è così lontana e la ferita non è cicatrizzata: i collaboratori del giornale hanno visto questa guerra. Petru Rocca era ufficiale ed è stato colpito; ha ricevuto la Croce di guerra con le palme e la Legion

\section{Per quella chi s'annega}

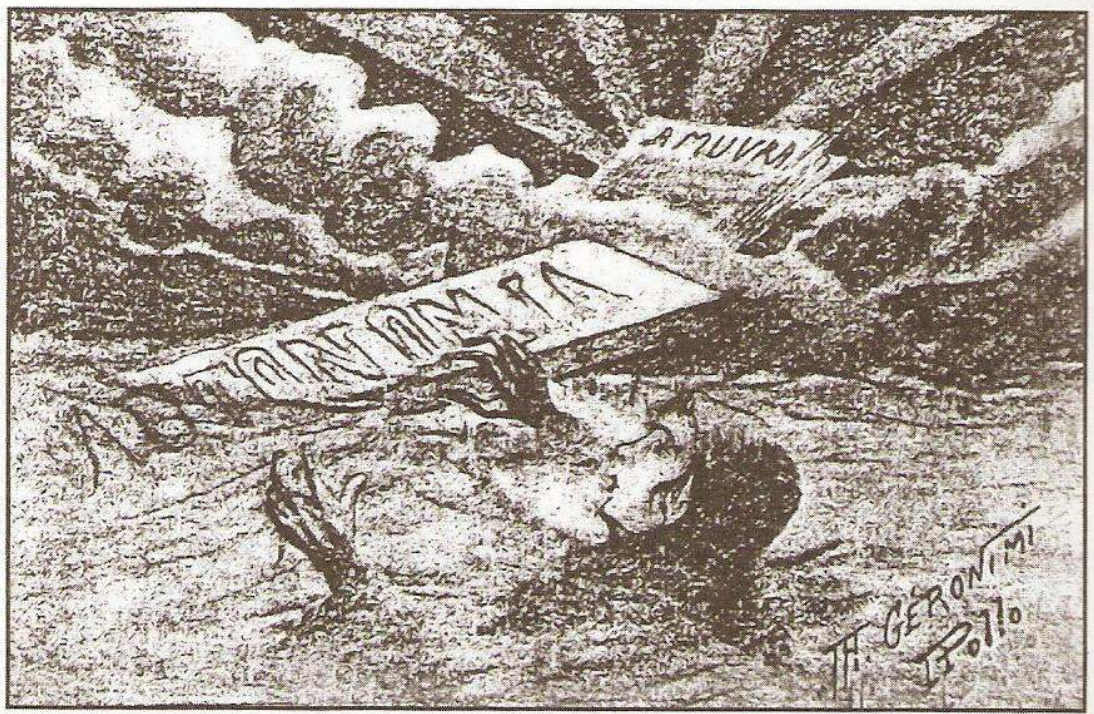

A Tavula di Salvezza

J.A.Geronimi e P. Pollo, A Muvra, 13 giugno 1926

d'onore.

Inoltre i corsisti desiderano l'autonomia amministrativa della Corsica e non la sua autonomia militare.

Le caricature non danno conto dell'emigrazione femminile. Come ricorda Janine

Renucci: «C'est après 1920 que grossit la vague d'émigration masculine et que se déclenche l'émigration féminine, qui finit par prédominer ${ }^{26}$.

Nel complesso la caricatura non si accontenta di denunciare una situazione: l'opinione pubblica è così allarmata che è allora possibile farla aderire alle soluzioni che le vengono proposte per far uscire l'isola dalla sua decadenza. È necessario resistere. La soluzione viene esposta in maniera chiara, non con parole mascherate o immagini codificate in senso enigmatico, sebbene sia, dal 1921 suggerita da un sol levante, simbolo della speranza dei giorni migliori, e da un muflone, simbolo della lotta della Corsica per l'autonomia. Dal 1921 al 1937 ben trentotto disegni evocano la resistenza. I

${ }^{26}$ RENUCCI, Janine, Corse traditionnelle et Corse nouvelle, Lyon, Audin impr., 1974, p. 136. 
disegnatori insistono a tal punto sul degrado economico e sociale dell'isola dopo la sua integrazione alla Francia, sull'incuria da parte del governo e sul non-senso storico di questa integrazione, che ha terminato una perdita della sua identità, al punto che la sola soluzione possibile sembra effettivamente essere l'autonomia. La parola autonomia appare sul testo della rivista dal 1920, prodotto della penna di Petru Rocca, ma il contenuto di questo concetto è ancora impreciso. Nel corso degli anni s'affina per affermarsi definitivamente nel 1926, data in cui la parola autonomia appare per la prima volta in una caricatura di J. A. Geronimi e P. Pollo: la Corsica parte alla deriva, ma rimane un'ancora di salvezza. Tuttavia, se questa parola pure è espressa, questo disegno non ci indica in alcun modo il contenuto di questo termine. I disegni sono quelli tipici degli ultimi anni. Questo testimonia che il movimento ha avuto bisogno di una certa maturità per arrivare a definire la sua posizione, dopo averla ricercata. A partire dal 1926 venticinque disegni ostentano la parola autonomia, di cui sette solo nell'anno 1937.

Per giustificare le loro rivendicazioni, i corsisti si riallacciano a Pascal Paoli. Questo

CI VOLE' A CASCACCI

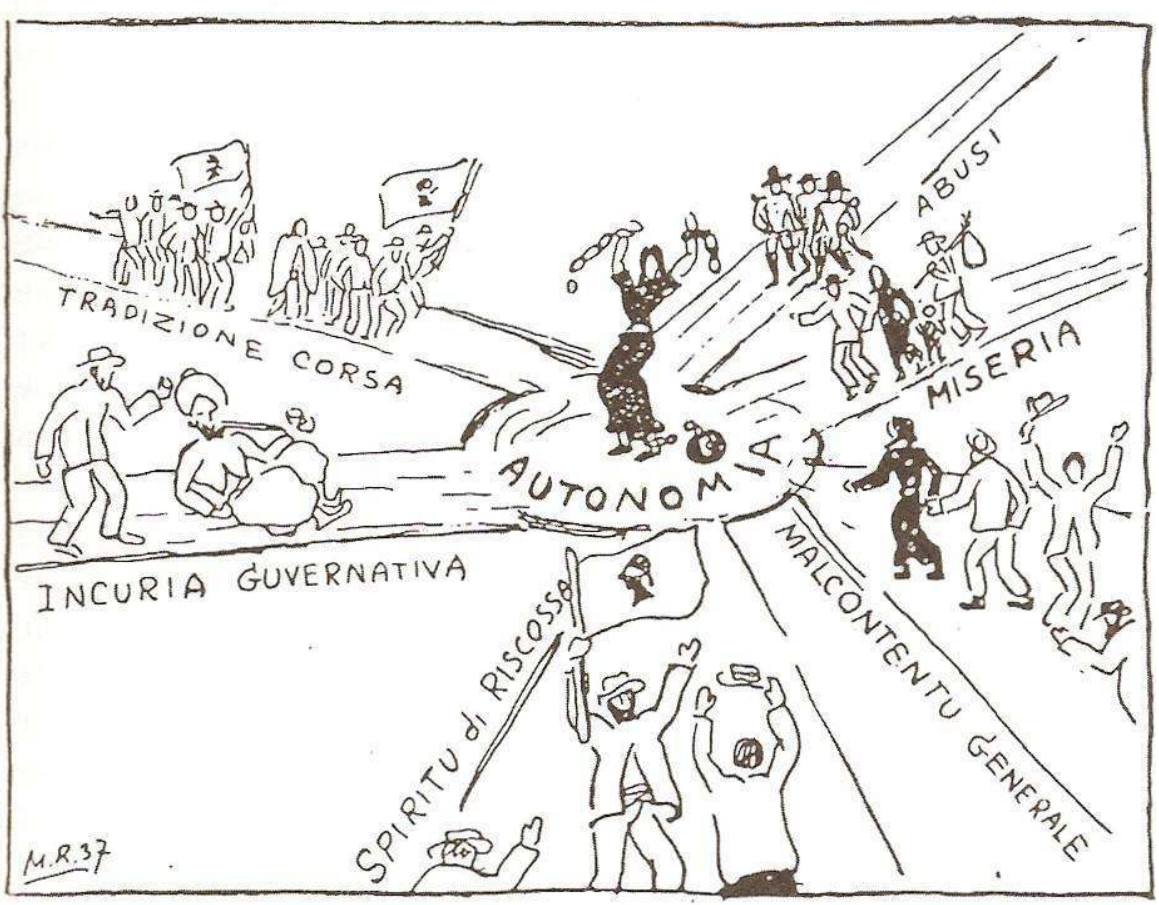

TUTTE E STRADE CUNDUCENU A I'AUTONOMIA

Matteo Rocca, A Muvra, 6 giugno 1937. periodo è considerato come il più glorioso: il ricordo del Babbu è esaltato. Diciannove disegni vi fanno riferimento.

Per questo motivo è importante situare questo dibattito nel contesto internazionale dell'epoca. L’inizio del secolo è marcato dall'affermazione di un sentimento nazionale in seno al quale si sviluppano movimenti di protesta 
che rivelano, per riprendere il termine di Pierre Renouvin, minoranze nazionali ${ }^{27}$. È incontestabile che, negli ultimi anni del XIX secolo e i primi del XX, la protesta delle minoranze nazionali acquisisca, pressoché dappertutto in Europa, una nuova grandezza. Le forze che determinano questi movimenti sono talvolta legate alla situazione economica e sociale, ma sono soprattutto di ordine ideale: volontà di salvaguardare un sistema d'idee, di tradizioni, di credenze religiose; volontà di conservare il diritto di esprimersi nella propria lingua materna.

La stampa prende parte a questo risveglio; è attraverso questa che i movimenti di protesta si esprimono. La Corsica partecipa a questa grande corrente, che attraversa tutta l'Europa.

Malgrado ciò i corsisti si difendono dall'accusa di essere separatisti e la caricatura è orientata in tal senso. Daniel Polacci precisa che, a suo parere, è tra il 1932 e il 1937: «que A Muvra devient réellement irrédentiste, non plus seulement en tendance, mais consciemment et presque ouvertement» ${ }^{28}$. Dal 1932, otto disegni, su poco più di una decina, fanno riferimento all'irredentismo; ma, ed è un paradosso, le illustrazioni precedenti a questa data sono le più esplicite. Anche se i disegnatori preferiscono fare ricorso alla derisione o alla provocazione, è lo humour che domina. è un modo di approcciare un tema imbarazzante e di deviarne l'attenzione, dal momento che i corsisti erano sospettati d'irredentismo: per questo verso, questi ultimi non rispondono esplicitamente agli interrogativi. $\mathrm{O}$ il non rispondere è, forse, un modo di confessare?

\section{Conclusioni}

Così, la caricatura politica sulla rivista A Muvra non rispecchia le testimonianze scritte. Sono illustrati i temi ricorrenti, come la povertà dell'isola, l'emigrazione dei suoi abitanti, il numero rilevante dei funzionari corsi o la nascita di un movimento regionalista poi autonomista. Ma la caricatura non è solamente il pallido riflesso dei documenti scritti: va ben oltre. In effetti, poiché è una caricatura politica di propaganda, orienta l'immagine della Corsica e dei Corsi verso una visione più conforme alle loro preoccupazioni. Gradualmente, a piccoli passi, in modo sornione, i disegnatori insistono su alcuni aspetti negandone altri. Così accentuano l'idea dell'abbandono dell'isola da parte dello Stato francese, mettendo in secondo piano la

27 RENOUVIN, Pierre, Le Mouvement des nationalités en Europe dans la seconde moitié du XIXe siècle, 3 voll., Paris, Centre de documentation universitaire, 1946 [n.d.t.].

28 POLACCI, Daniel, Les autonomistes corses de l'entre-deux-guerres, Mémoire de Maîtrise, Aix en Provence, 1974, p. 146. 
sua povertà: non inventano nulla, ma "creano degli squilibri". Questi "aggiustamenti" sono difficilmente percepibili per i lettori dal momento che si fondano su realtà che nessuno contesta.

La caricatura illustra e completa il messaggio politico dei corsisti, che reclamano l'autonomia dell'isola e il ritorno alle tradizione e alla lingua dei maquis. La loro lotta è giustificata dalla loro storia: l'epoca di Pascal Paoli, quando la Corsica era indipendente, viene considerata come l'epoca d'oro a cui bisogna fare ritorno.

Per essere comprensibile la caricatura deve riassumere il suo messaggio e andare all'essenziale: circoscrivere un bersaglio implica l'operare delle scelte. La caricatura restituisce un'immagine incompleta e orientata: pecca di omissione. Insiste a tal punto sull'incuria del governo che la soluzione dell'autonomia che propone appare come un'evidenza e sembra effettivamente essere la migliore, se non l'unica.

La caricatura pecca così di esagerazione. Dal momento che è un documento iconografico, impone un'immagine stereotipata della "vera Corsica". L’immaginazione del lettore non è messa alla prova, dal momento che i disegni rappresentano fisicamente gli isolani e gli "altri". Ispirandosi a correnti d'opinione, come la definizione d'una razza o la difesa di una minoranza nazionale, la caricatura impone dei clichés. Introduce la nozione di una "superiorità della razza" corsa in rapporto alle altre. Una volta di più organizza l'opinione pubblica e prova ad influenzarne i comportamenti.

$\mathrm{Ma}$, al contempo, la caricatura restituisce l'immagine di una Corsica ancorata al suo passato e alle sue tradizioni: dà l’impressione di rifiutare ogni modernismo, che rischia di intaccarla e di farle perdere la sua identità. Questo genera una forma di paradosso: da una parte denuncia la mancanza d’investimenti finanziari e di interesse da parte della Francia, ma anche, sistematicamente, tutto ciò che viene dal continente come la fonte della perdita dell'anima corsa.

Infine la peculiarità di una caricatura è di essere effimera. È vero che alcuni disegni hanno oggi perso il loro valore umoristico, appaiono persino troppo duri, ma non si può dubitare del fatto che risultassero molto divertenti. È dunque un paradosso se molte illustrazioni ci fanno ancora sorridere, se non ridere apertamente, e sembrano essere ancora molto attuali... 


\section{* L'autore}

Marie-Claude Lepeltier è nata nel 1962 nella regione della Basse-Normandie. Giunta in Corsica, ha ripreso i suoi studi storia conseguendo la laurea nel 1997, presentando una tesi dal titolo: Image de la Corse et des Corses à travers la caricature insulaire sous la IIIe République. Attualmente è bibliotecaria dell'Université Pascal Paoli di Corté. Responsabile del fondo regionale, lavora all'attuazione di un "piano di conservazione condivisa dei periodici corsi" in collaborazione con le biblioteche pubbliche e gli Archivi dipartimentali dell'isola.

URL: < http://www.studistorici.com/progett/autori/\#Lepeltier >

\section{Per citare questo articolo:}

LEPELTIER, Marie-Claude, «"A Muvra”, 1920-1939: la caricatura in Corsica», Diacronie. Studi di Storia Contemporanea : Periferie. Cultura, economia, politica, 29/3/2014,

URL: < http://www.studistorici.com/2014/3/29/lepeltier_numero_17/ >

\section{Diacronie Studi di Storia Contemporanea $\beta$ www.diacronie.it}

Risorsa digitale indipendente a carattere storiografico. Uscita trimestrale. redazione.diacronie@hotmail.it

Comitato di redazione: Jacopo Bassi - Luca Bufarale - Elisa Grandi - Deborah Paci - Fausto Pietrancosta - Matteo Tomasoni - Luca Zuccolo 